\title{
Novo registro de Trichospilus diatraeae Cherian \& Margabandhu, 1942 (Hymenoptera: Eulophidae), como parasitóide de Spodoptera cosmioides Walker, 1858 (Lepidoptera: Noctuidae) no Brasil
}

\author{
Bruno Zaché ${ }^{1,2}$, Carlos Frederico Wilcken ${ }^{1}$, \\ Ronelza Rodrigues da Costa Zachél \& Natália Medeiros de Souza ${ }^{I}$ \\ ${ }^{1}$ Departamento de Proteção de Plantas, Faculdade de Ciências Agronômicas, \\ Universidade Estadual Paulista Julio Mesquita Filho - UNESP, CEP 18603-970, Botucatu, SP, Brasil \\ ${ }^{2}$ Autor para correspondência: Bruno Zaché, e-mail: bzache@bol.com.br
}

ZACHÉ, B., WILCKEN, C.F., ZACHÉ, R.R.C. \& SOUZA, N.M. New occurrence of Trichospilus diatraeae Cherian \& Margabandhu, 1942 (Hymenoptera: Eulophidae) as a parasitoid of Spodoptera cosmioides Walker, 1858 (Lepidoptera: Noctuidae) in Brazil. Biota Neotrop. 12(1): http://www.biotaneotropica.org.br/ v12n1/en/abstract?inventory+bn01212012012

\begin{abstract}
Spodoptera cosmioides (Lepidoptera: Noctuidae) is a polyphagous species and feeds on many wild and cultivated plants. In Brazil, the crops of pineapple, cotton, rice, eggplant, onions, eucalyptus, bell pepper and tomato, among other vegetables, are considered to be host of the pest. However, despite the wide host range, its occurrence as a pest is reported related to imbalances caused by excessive use of broad-spectrum insecticides, which is causing pest resistance to insecticides. Because of this complexity, alternative control methods have been proposed, for example, biological control using parasitoids. Trichospilus diatraeae (Hymenptera: Eulophidae) is a gregarious pupal parasitoid species, preferably from the order Lepidoptera. This is the first report of T. diatraeae parasitizing pupae of $S$. cosmioides, parasitoid offers new prospects for biological control programs.

Keywords: Spodoptera cosmioides, polyphagia, biological control, pupal parasitoid, Trichospilus diatraeae.
\end{abstract}

ZACHÉ, B., WILCKEN, C.F., ZACHÉ, R.R.C. \& SOUZA, N.M. Novo registro de Trichospilus diatraeae Cherian \& Margabandhu, 1942 (Hymenoptera: Eulophidae), como parasitóide de Spodoptera cosmioides Walker, 1858 (Lepidoptera: Noctuidae) no Brasil. Biota Neotrop. 12(1): http://www.biotaneotropica.org.br/ v12n1/pt/abstract?inventory+bn01212012012

Resumo: Spodoptera cosmioides (Lepidoptera: Noctuidae)é uma espécie polífaga e alimenta-se de grande número de plantas cultivadas e espontâneas. No Brasil, as culturas do abacaxi, algodoeiro, arroz, berinjela, cebola, eucalipto, pimentão e tomateiro, entre outras hortaliças, são consideradas hospedeiras da praga. Entretanto, apesar da ampla gama de hospedeiros, sua ocorrência como praga é relatada relacionada a desequilíbrios provocados pelo uso excessivo de inseticidas de amplo espectro, o que vem causando a resistência da praga a inseticidas. Devido a essa complexidade, métodos alternativos de controle têm sido propostos, por exemplo, o controle biológico através da utilização de parasitóides. Trichospilus diatraeae (Hymenptera: Eulophidae) é um parasitóide gregário pupal preferencialmente de espécies da ordem Lepidoptera. Este é o primeiro relato de T. diatraeae parasitando pupas de $S$. cosmioides, parasitóide que oferece novas perspectivas para os programas de controle biológico.

Palavras-chave: Spodoptera cosmioides, polifagia, controle biológico, parasitoide de pupas, Trichospilus diatraeae. 


\section{Introdução}

Trichospilus diatraeae é um parasitoide pupal gregário, preferencialmente de insetos da Ordem Lepidoptera (Boucek 1976). Sua presença já foi constatada nas famílias: Crambidae (Cherian e Margabanghu, 1942), Noctuidae (Etienne e Viette, 1973, Zaché et al., 2010), Pyralidae, (Etienne e Viette, 1973, Boucek, 1976, Bennett et al. 1987), Nymphalidae (Boucek, 1976), Geometridae (Bennett et al. 1987), Pieridae (Torres-Bauza, 1994), Arctiidae (Paron e Berti-Filho, 2000), e Oecophoridae (Oliveira, et al., 2005).

Em 1963, T. diatraeae foi da Índia para Trinidad e Tobago (Antilhas) e para os EUA utilizando como hospedeiros Diatraea spp. (Pyralidae) (Bennett et al. 1987). Bordat et al. (1977) realizaram liberações de $T$. diatraeae para controle de pragas de gramíneas no Senegal (África), utilizando o hospedeiro Chilo suppressalis Walker (Pyralidae). Etienne \& Viette (1973) e Etienne (1975) relataram T. diatraeae, nas Ilhas Reunião e Maurício, parasitando as pragas da cana-de-açúcar Melanitis leda L. e Hedylepta indicata F. (Pyralidae) e Polydesma umbricola Boisduval (Noctuidae) praga da conífera Trichopolydesma collutrix. Bournier (1975) utilizou este parasitoide no controle biológico de noctuídeos pragas de algodão em Madagascar (África), utilizando como hospedeiro Anagasta kuehniella Zell. Em Comores, Brenière et al. (1985) criaram e liberaram de $T$. diatraeae, na tentativa de controlar Chilo partellus Swinhoe (Pyralidae), praga do milho. Rodriguez-Del-Bosque \& Smith Junior (1989) realizaram testes com T. diatraeae, nos EUA para controle da broca do milho Diatraea lineolata Walker (Pyralidae). Betbeder-Matibet (1990) estudou a criação massal de Chilo sacchariphagus para a obtenção de T. diatraeae. Torres-Bauza (1994) registrou o parasitoide atacando pupas de Dismorphia spio (Pieridae) em Ingá vera (Mimosidae) em Porto Rico (Antilhas).

No Brasil, a introdução de $T$. diatraeae teria sido acidental e sua primeira ocorrência teria sido reportada em 1996 em pupas de Arctiidae (Lepidoptera) na cidade de Piracicaba no estado de São Paulo (Paron \& Berti Filho 2000). Em relação a pragas florestais T. diatraeae foi coletado em pupas de Thyrinteina arnobia em plantio de eucalipto no estado de Minas gerais (Pereira et al. 2008). Em 2010 foi reportado o parasitismo de $T$. diatraeae em pupas da lagarta desfolhadora do eucalipto Melanolophia consimilaria (Walker) (Lepidoptera: Geometridae) uma praga que pupa no solo e Hypsipyla grandella família Pyralidae praga de Swietenia macrophylla (Zaché et al. 2010a,b), Em 2011 se descreveu sua ocorrência
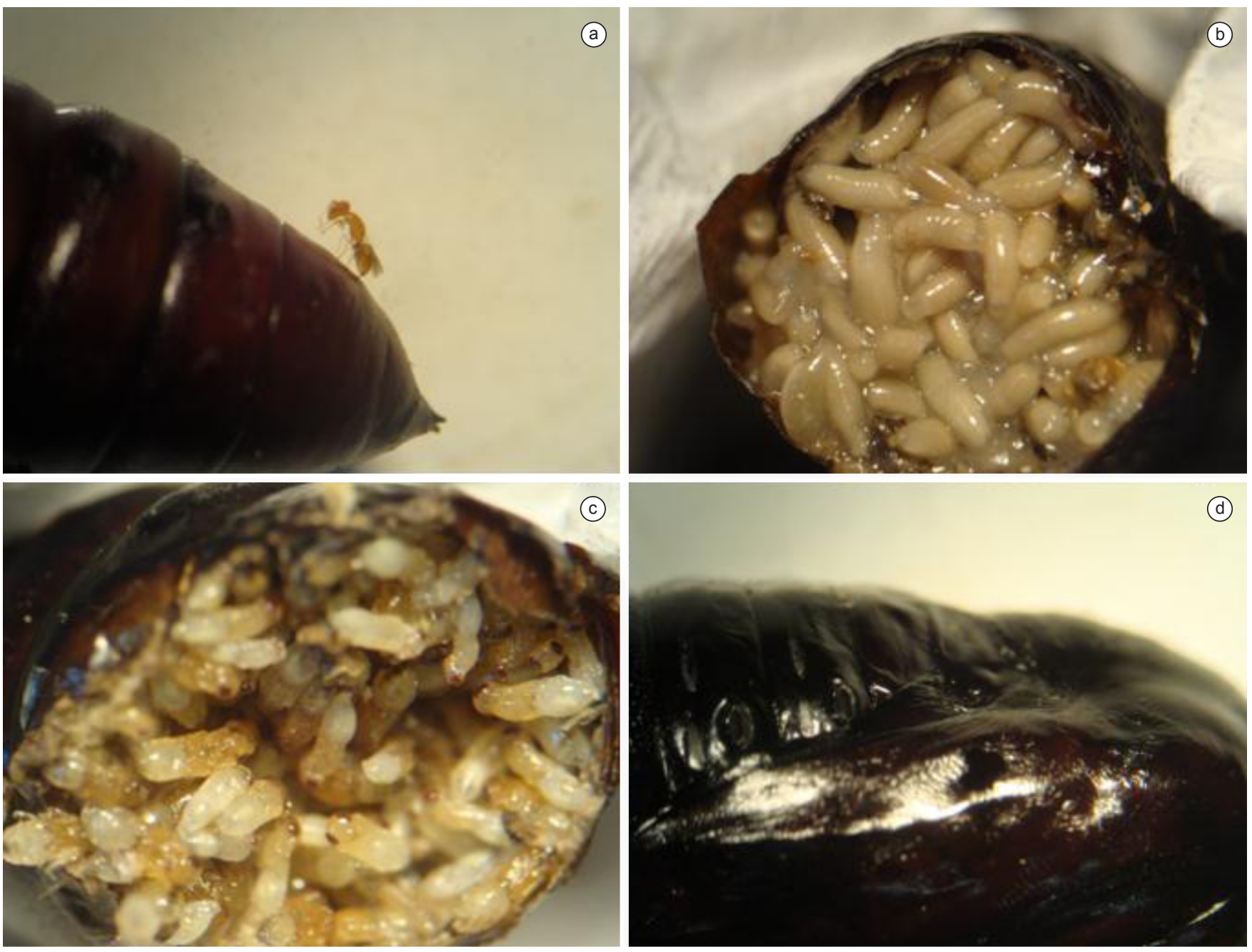

Figura 1. Fêmea de Trichospilus diatraeae (Hymenoptera: Eulophidae) (a); Larvas do parasitoide dentro da pupa de Spodoptera cosmioides (Lepidoptera: Noctuidae) (b); Pupas do parasitoide (c); Orificio de emergência de T. diatareae (d).

Figure 1. Female Trichospilus diatareae (Hymenoptera: Eulophidae) (a); the parasitoid larvae in Spodoptera cosmioides (Lepidoptera: Noctuidae) (b); the parasitoid pupae (c); emergence hole of $T$. diatraeae (d). 
em Euselasia eucerus, uma espécie da família Riodinidae e Sarcina violascens (Lepdoptera: Limantriidae) (Zaché et al. 2011a,b).

Spodoptera cosmioides (Walk.) é uma espécie polífaga e alimenta-se de grande número de plantas cultivadas e espontâneas. No Brasil, as culturas do abacaxi (mudas), algodoeiro (maçãs), arroz, berinjela, cebola (partes verdes), eucalipto (mudas), mangueira, pimentão e tomateiro, entre outras hortaliças, são consideradas hospedeiras da praga (Bertels 1953, Silva et al. 1968, Santos et al. 1980).

De acordo com Silvain \& Lalanne-Cassou (1997) e Lalanne-Cassou et al. (1999), S. cosmioides era considerada sinonímia de Spodoptera latifascia (Walk.). Entretanto, esses autores demonstraram a existência de diferenças moleculares, morfológicas, fisiológicas e comportamentais entre elas, considerando-as espécies distintas. Ambas são membros de um complexo de espécies neotropicais, sendo que S. latifascia está estabelecida na América Central, Antilhas e sul do EUA enquanto $S$. cosmioides é encontrada na América do Sul.

Entretanto, apesar da ampla gama de hospedeiros, sua ocorrência como praga é relatada relacionada a desequilíbrios provocados pelo uso excessivo de inseticidas de amplo espectro, o que vem causando a resistência da praga a inseticidas. Devido a essa complexidade, métodos alternativos de controle têm sido propostos, como por exemplo, o controle biológico (Habib et al. 1983).

$\mathrm{O}$ objetivo deste estudo foi avaliar o impacto de T. diatraeae como parasitóide de pupas de $S$. Cosmioides praga altamente polifaga, como uma alternativa potencial para o controle químico.

\section{Material e Métodos}

Parasitóides utilizados neste teste foram originalmente coletadas em pupas de Iridopsis sp. (Lepidoptera: Geometridae) em plantios de eucalipto no município de Curvelo, Minas Gerais, Brasil, em 2009, enviados a Dr Valmir Antonio Costa no Instituto Biológico, Campinas, São Paulo para identificação e armazenamento como voucher. Posteriormente, em laboratório foi mantida, com pupas de Spodoptera frugiperda (Stoll) (Lepidoptera: Noctuidae) como hospedeiro. A técnica de criação do parasitóide foi descrita em detalhes por Zaché et al. (2010a).

As pupas de $S$. cosmioides foram coletadas na criação estoque, sendo criadas em condições controladas sobre dieta artificial descrita por Giolo et al. (2002), onde 15 pupas com 24 horas de formadas foram oferecidas às fêmeas de $T$. diatraeae com 24 horas de emergência na proporção 28:1 (parasitóide: hospedeiro) por um período de 72 horas. A partir daí, a fêmea foi retirada e as pupas foram mantidas em tubos de vidro $(2,5 \mathrm{~cm}$ de diâmetro e $8,5 \mathrm{~cm}$ de comprimento) vedados com tecido tipo "voil" e mantidas em câmara climática a $26 \pm 2{ }^{\circ} \mathrm{C}, 60 \pm 10 \%$ de umidade relativa e fotofase de 12 horas. Os parâmetros avaliados foram: nível de parasitismo, o número de parasitoides emergidos e não emergidos, a duração do ciclo ovo-adulto. As pupas de $S$. cosmioides foram dissecadas para avaliar os parasitóides não emergidos.

\section{Resultados e Discussão}

Os parasitoides alcançaram taxas de parasitismo de $100 \%$, com uma taxa de emergência de 100\%. Uma média de 868,4 ( $\pm 0,53 \mathrm{EP})$ parasitóides / pupa emergiram enquanto apenas $0,4( \pm 0,03 \mathrm{EP})$ parasitóides por pupa não conseguiram emergir. $\mathrm{O}$ ciclo de ovo a adulto de $T$. diatraeae foi de $31,3( \pm 0,97 \mathrm{EP})$ dias.

Estes resultados demonstram pela primeira vez em condições controladas a capacidade de $T$. diatraeae de parasitar pupas de S. cosmioides, um Lepidoptera da família Noctuidae (Figura 1). Novos estudos são necessários para determinar o potencial de T. diatraeae para o controle biológico dessa praga em campo, podendo levar a uma redução no uso indiscriminado de inseticidas químicos para o controle dessa praga sujeita a uma ampla gama de pesticidas.

\section{Referências Bibliográficas}

BENNETT, F.D., GLENN, H., YASEEN, M. \& BARANOWSKI, R.M. 1987. Records of Trichospilus diatraeae, an Asian parasite (Hymenoptera: Eulophidae) from the Caribbean and Florida. Fla. Entomol.. 70:184-186. http://dx.doi.org/10.2307/3495110

BERTELS, A. 1953. Pragas de solanáceas cultivadas. Agros. 6:154-160.

BETBEDER-MATIBET, M. 1990. Élevage de plusieurs espéces du genre Chilo et de certains de leurs parasites pour la lutte biologique contre les foreurs des graminees en Afrique. Insect Sci. Appl. 11:617-623.

BORDAT, D., BRENIERE, J. \& COQUARD, J. 1977. Foreurs de gramines africaines: parasitisme et techniques d'élevage. Agron. Trop. 32:391-399.

BOUČEK, Z. 1976. The African and Asiatic species of Trichospilus and Cotterellia (Hymenoptera: Eulophidae). Bull. Entomol. Res. 65:669-681. http://dx.doi.org/10.1017/S0007485300006362

BOURNIER, J.P. 1975. Sur la reproduction parthenogenetique de Trichosplilus diatraeae Cher. et Margab. (Hymenoptera: Chalcidoidea). Bull. Soc. Entomol. France. 80:116-118.

BRENIERE, J., BORDAT, D., VERCAMBRE, B., HAMZA, H. \& RENAND, M. 1985. Les opérations de lutte biologique contre lê foreur du maïs Chilo partellus (Swinhoe), Lepidoptera, dans L'île de Ngazidja. Agron. Trop. 40:157-166.

CHERIAN, M.C. \& MARGABANDHU, V. 1942. A new species of Trichospilus (Hymenoptera: Chalcidoidea) from South India. Indian Journal of Entomology. 4:101-102.

ÈTIENNE, J. 1975. Notes sur l'élevage et la biologie de Pseudoperichaeta laevis (Diptera: Tachinidae) sur hote de laboratoire. Entomophaga. 20:105111. http://dx.doi.org/10.1007/BF02373456

ÈTIENNE, J. \& VIETTE, P. 1973. Nouvelle note sur Polydesma umbricola Boisduval (Lepidoptera: Noctuidae). Bull. Soc. Entomol. France. 78:98107.

GIOLO, F.P., GRÜTZMACHER, A.D., GARCIA, M.S. \& BUSATO, G.R. 2002. Parâmetros biológicos de Spodoptera frugiperda (J.E. Smith, 1797) (Lep.: Noctuidae) oriundas de diferentes localidades e hospedeiros. Rev. Bras. Agrocienc. 8(3):219-224.

HABIB, M.E.M., PALEARI, L.M. \& AMARAL, M.E.C. 1983. Effect of three larval diets on the development of the armyworm, Spodoptera latifascia Walker, 1856 (Noctuidae, Lepidoptera). Rev. Bras. Zool. 1:177-182.

LALANNE-CASSOU, B., SILVAIN, J.F., MONTI, L. \& MALOSSE, C. 1999. Mecanismes d'isolement reproducteur chez les especes du complexe neotropical Spodoptera latifascia - S. cosmioides - S. descoinsi (Lepidoptera: Noctuidae). Actes de la IV Conference Internationale Francophone d'Entomologie. Saint-Malo, France, 5-9 juillet, 1998. Ann. Soc. Entomol. Fr. 35:109-116.

OLIVEIRA, H.N., ZANUNCIO, J.C., PEDRUZZI, E.P. \& ESPINDULA, M.C. 2005. Rearing of Thyrinteina arnobia (Lepidoptera: Geometridae) on guava and eucalyptus in laboratory. Brazilian Braz. Arch. Biol. Technol. 48:801-806. http://dx.doi.org/10.1590/S151689132005000600016

PARON, MR. \& BERTI-FILHO, E. 2000. Capacidade reprodutiva de Trichospilus diatraeae (Hymenoptera: Eulophidae) em pupas de diferentes hospedeiros (Lepidoptera). Scientia. Agricola. 57:355-358. http://dx.doi.org/10.1590/S0103-90162000000200025

PEREIRA, F.F., ZANUNCIO, J.C., TAVARES, M.T., PASTORI, P.L. \& JACQUES, G.C. 2008. Record of Trichospilus diatraeae (Hymenoptera: Eulophidae) as parasitoid of the eucalypt defoliator Thyrinteina arnobia (Lepidoptera: Geometridae) in Brazil. Phytoparasitica. 36:304-306. http:// dx.doi.org/10.1007/BF02980777 
Zaché, B. et al.

RODRIGUEZ-DEL-BOSQUE, L.A. \& SMITH JUNIOR, J.W. 1989. Parasitization of Diatraea lineolata pupa and diapausing larvae by several exotic parasites. Fla. Entomol. 72:703-705. http://dx.doi. org/10.2307/3495051

SANTOS, G.P., COSENZA, G.W. \& ALBINO, J.C. 1980. Biologia de Spodoptera latifascia (Walker, 1856) (Lepidoptera: Noctuidae) sobre folhas de eucalipto. Rev. Bras. Entomol. 24:153 155.

SILVA, A.G.A., GONÇALVES, C.R., GALVÃO, D.M., GONÇALVES, A.J.L., GOMES, J., SILVA, M.N. \& SIMONI, L. 1968. Quarto catálogo dos insetos que vivem nas plantas do Brasil, seus parasitos e predadores. Rio de Janeiro, Ministério da Agricultura, Tomo 1, Parte II

SILVAIN, J.F. \& LALANNE-CASSOU, B. 1997. Distinction entre Spodoptera latifascia (Walker) et Spodoptera cosmioides (Walker), bona species (Lepidoptera: Noctuidae). Rev. Franc. d'Entomol. 19:95-97.

TORRES-BAUZA, J.A. 1994. Hymenopterous parasitoids of Dismorfia spio (Pieridae: Dismorphiinae). J. Lepid. Soc. 48:266.
ZACHÉ, B., WILCKEN, C.F., DACOSTA, R.R., SOLIMAN, E.P. 2010a. Trichospilus diatraeae Cherian \& Margabandhu, 1942 (Hymenoptera: Eulophidae), a new parasitoid of Melanolophia consimilaria (Lepidoptera: Geometridae). Phytoparasitica. 38:355-357. http://dx.doi.org/10.1007/ s12600-010-0108-6

ZACHÉ, B., WILCKEN, C.F., ZACHÉ, R.R.C., SOLIMAN, E.P. \& SAN ROMAN, M.L.L. 2010b. Trichospilus diatraeae Cherian \& Margabandhu, 1942 (Hymenoptera: Eulophidae), un nuevo parasitoide de Hypsipyla grandella (Zeller, 1848) (Lepidoptera: Pyralidae). Idesia. 28:111-114.

ZACHÉ, B., ZACHÉ, R.R.C.; SOLIMAN, E.P. \& WILCKEN, C.F. $2011 \mathrm{a}$. Evaluation of Trichospilus diatraeae (Hymenoptera: Eulophidae) as parasitoid of the eucalyptus defoliator Euselasia eucerus (Lepidoptera: Riodinidae). Int. J. Trop. Insect Sci. 20:1-5.

ZACHÉ, B., ZACHÉ, R.R.C., SOUZA, N.M.; DIAS, T.K.R. \& WILCKEN, C.F. 2011b. New record of Trichospilus diatraeae margabandhu \& cherian, 1942 (hymenoptera: eulophidae) parasitizing Sarsina violascens (herrich-schaeffer, 1856) (lepidoptera: lymantriidae) in brazil. J. Plant. Protect. Res. 51:420-422.

Recebido em 08/05/2011

Versão reformulada recebida em: 04/02/2012

Publicado em 23/02/2012 